\title{
Screening of Compounds for Anti-tuberculosis Activity, and in vitro and in vivo Evaluation of Potential Candidates
}

OPEN ACCESS

Edited by:

Divakar Sharma

University of Delhi, India

Reviewed by:

Diana Machado,

New University of Lisbon, Portugal

Kaushiki Mazumdar,

Kelly Government Solutions,

United States

Priyanka Mishra,

University of Texas at San Antonio,

United States

*Correspondence:

Zhen F. Fu

zhenfu@uga.edu

Xi Chen

chenxi419@mail.hzau.edu.cn

Gang Cao

gcao@mail.hzau.edu.cn

tThese authors have contributed equally to this work and share first authorship

Specialty section:

This article was submitted to Antimicrobials, Resistance

and Chemotherapy,

a section of the journal

Frontiers in Microbiology

Received: 26 January 2021 Accepted: 26 May 2021

Published: 30 June 2021

Citation:

Zhou W, Yang B, Zou Y, Rahman K, Cao X, Lei Y, Lai R, Fu ZF, Chen $X$ and Cao $G$ (2021) Screening of Compounds for Anti-tuberculosis Activity, and in vitro and in vivo Evaluation of Potential Candidates. Front. Microbiol. 12:658637. doi: 10.3389/fmicb.2021.658637

\author{
Wei Zhou ${ }^{1,2+}$, Bing Yang ${ }^{1,2+}$, Yanyan Zou',2, Khaista Rahman ${ }^{1,2}$, Xiaojian Cao ${ }^{1,2}$, \\ Yingying Lei ${ }^{1,2}$, Ren Lai ${ }^{3}$, Zhen F. Fu' ${ }^{1,2 *}, X_{i}$ Chen ${ }^{1,2 *}$ and Gang Cao ${ }^{1,2,4,5 *}$
}

\begin{abstract}
${ }^{1}$ State Key Laboratory of Agricultural Microbiology, Huazhong Agricultural University, Wuhan, China, ${ }^{2}$ College of Veterinary Medicine, Huazhong Agricultural University, Wuhan, China, ${ }^{3}$ Key Laboratory of Animal Models and Human Disease Mechanisms, Chinese Academy of Sciences and Yunnan Province, Kunming Institute of Zoology, Kunming, China, ${ }^{4}$ Bio-Medical Center, Huazhong Agricultural University, Wuhan, China, ${ }^{5}$ Cooperative Innovation Center for Sustainable Pig Production (CICSPPS), Huazhong Agricultural University, Wuhan, China
\end{abstract}

Tuberculosis (TB) is a debilitating infectious disease responsible for more than one million deaths per year. The emergence of drug-resistant TB poses an urgent need for the development of new anti-TB drugs. In this study, we screened a library of over 4,000 small molecules and found that orbifloxacin and the peptide AK15 possess significant bactericidal activity against Mycobacterium tuberculosis (Mtb) in vitro. Orbifloxacin also showed an effective ability on the clearance of intracellular Mtb and protect mice from a strong inflammatory response but not AK15. Moreover, we identified 17 nucleotide mutations responsible for orbifloxacin resistance by whole-genome sequencing. A critical point mutation (D94G) of the DNA gyrase (gyrA) gene was found to be the key role of resistance to orbifloxacin. The computational docking revealed that GyrA D94G point mutation can disrupt the orbifloxacin-protein gyrase interactions mediated by magnesium ion bridge. Overall, this study indicated the potential ability of orbifloxacin as an anti-tuberculosis drug, which can be used either alone or in combination with first-line antibiotics to achieve more effective therapy on TB.

Keywords: Mycobacterium tuberculosis, orbifloxacin, drug resistance, DNA gyrase, molecular docking, tuberculosis, combination therapy

\section{INTRODUCTION}

Tuberculosis (TB), caused by Mycobacterium tuberculosis (Mtb), is a life-threatening infectious disease, which was responsible for 1.3 million deaths globally in 2020 [World Health Organization (WHO) (2020)]. The World Health Organization reported that approximately 23\% (1.7 billion) of the world's population has been latently infected by $M t b$, and 1.5 million people develop active TB every year. The treatment regimen for TB mainly comprises antibiotics such as rifampicin (RIF), isoniazid (INH), ethambutol, pyrazinamide, and aminoglycosides (Fatma et al., 2020). Therapy

Abbreviations: TB, tuberculosis; Mtb, Mycobacterium tuberculosis; RIF, rifampicin; INH, isoniazid; MDR, multiple drugresistant; XDR, extensively drug-resistant; gyrA, DNA gyrase subunit A; MIC, minimum inhibitory concentration; CFU, colony-forming unit; PBS, phosphate-buffered saline; FIC, fractional inhibitory concentration. 
with these antibiotics must be taken for 6 months to 2 years, which can result in the emergence of drug resistance and poor therapeutic outcomes (Adams et al., 2011; Akos et al., 2015; Castro et al., 2020). With the emergence of multidrug-resistant (MDR) and extensively drug-resistant (XDR) strains (Tao et al., 2017; Arthur et al., 2019), tackling the disease using currently available therapeutic regimens is a huge challenge (Koul et al., 2011; Huynh and Marais, 2019; Fuad et al., 2020). Therefore, there is a desperate need for novel, effective anti-TB drugs that can render the bacteria more susceptible to treatment.

Over the last few decades, numerous attempts have been made to develop drugs to combat drug-resistant strains of $M t b$ (Soutter et al., 2016; Jansen and Rhee, 2017; Mosaei et al., 2018). Genes essential for mycobacterial growth (Sabine et al., 2015; Zuniga et al., 2015), enzymes associated with fatty acid synthesis (Tiago et al., 2019), and DNA replication have been targeted for new treatment options (Aggarwal et al., 2017; Pradhan and Sinha, 2018). However, clinical investigations revealed that the main mechanisms of drug resistance in $M t b$ are gene mutations and biofilm formation (Poushali et al., 2021; Saba et al., 2021). Therefore, screening of natural products and their derivatives (Guzman et al., 2012), new small molecule inhibitors (Mishra et al., 2018), and FDA-approved drugs offers a new approach for the development of novel anti-TB drugs (Igarashi, 2017; Luo et al., 2017; Igarashi et al., 2018; Nadav et al., 2018). Virtual screening using docking programs has been used to identify potential drug molecules from a virtual library containing 461,397 compounds. Following this, the biological activity of the identified molecules has been evaluated in vivo in a zebrafish model (Adams et al., 2011; Takaki et al., 2012).

In recent years, quinolones (Kumar et al., 2009) have been widely used in clinical treatment because of their high efficiency and broad-spectrum antibacterial activity (Grossman et al., 2014; Peraman et al., 2016; Correia et al., 2017; Samiksha et al., 2019). A therapeutic regimen using moxifloxacin, a fluoroquinolone derivative, has been developed for the treatment of patients with MDR and XDR-TB (Huynh and Marais, 2019; Nunn et al., 2019). However, the mechanism of action and details of the drug-Mtb interactions remain elusive.

In this study, we screened a library of over 4,000 small molecules, including natural products, FDA-approved drugs, and peptides, for compounds exhibiting activity against $M t b$. Orbifloxacin, the third generation of fluoroquinolone, was found to show potent anti-Mtb activity, both in vitro and in vivo, by targeting DNA gyrase subunit A (GyrA). Furthermore, we identified the mutations GyrA D94G and molecular mechanisms underlying orbifloxacin resistance.

\section{MATERIALS AND METHODS}

\section{Ethics Statements}

This study was carried out in accordance with the standards set by the Administration of Animal Care and Usage for Research and the Ministry of Health of China. Animals were housed in a specific-pathogen-free environment at the laboratory animal center of Huazhong Agricultural University. All experiments were approved by the Committee on the Ethics of Animal Experiments of the College of Veterinary Medicine, Huazhong Agricultural University (HZAUMO-2019-038).

\section{Bacterial Strain and Culture Conditions}

The Mtb H37Ra used in this study was obtained from the American Type Culture Collection (ATCC25177) and maintained in Difco Middlebrook 7H9 broth (Becton Dickinson), supplemented with $0.5 \%$ glycerol, $0.05 \%$ Tween 80 , and $10 \%$ oleic acid albumin dextrose catalase (OADC, BD, United States) at $37^{\circ} \mathrm{C}$. For bacterial culture and infection, all experiments were carried out in a biosafety level 2 laboratory following the appropriate biosafety standard operating procedures.

\section{Library of Drugs}

A total of 4,476 compounds from the natural products library, NIH clinical library, FDA-approved library, spectrum collection library, CYX library, LXD library, and peptide libraries were purchased from the Shanghai Institute of Materia Medica of the Chinese Academy of Sciences and Kunming. The compounds were aliquoted into 96-well microplates at a stock concentration of $10 \mathrm{mM}$. For drug screening, these compounds were diluted 10-fold with phosphate-buffered saline (PBS) to evaluate their bioactivity and safety.

\section{Microplate Alamar Blue Assay}

Mycobacterium tuberculosis $\mathrm{H} 37 \mathrm{Ra}$ was cultured to mid-log phase, with an optical density (OD) of $0.6\left(\sim 5 \times 10^{7}\right.$ colonyforming units $[\mathrm{CFU}] / \mathrm{ml}$ ) at $600 \mathrm{~nm}$. One hundred microliters $\left(5 \times 10^{4}\right.$ CFUs $)$ of bacterial suspension was added to each well of 96-well microplates. Then, $10 \mu \mathrm{M}$ of the compound was added to each well. DMSO was used as the negative control, and rifampicin (RIF) was used as the positive control. The plates were sealed and incubated at $37^{\circ} \mathrm{C}$ for 6 days. A $10 \%$ (v/v) solution of Alamar Blue was then added to each well. The anti-Mtb effect of each compound was determined based on the color change. The compound was considered to be inactive against $M t b$ if the color changed from blue to red.

\section{Determination of the Minimum Inhibitory Concentration of Selected Compounds}

For minimum inhibitory concentration (MIC) analysis, $100 \mu \mathrm{l}$ of the bacterial suspension was prepared as above and added to the wells of a 96-well plate. Ten microliters of twofold serial dilutions of screened drugs (from 0.012 to $12.8 \mu \mathrm{g} / \mathrm{ml}$ ) and peptides (from 1 to $512 \mu \mathrm{g} / \mathrm{ml}$ ) was added to the indicated well. Bacterial growth was assessed visually after culture for 1 week.

\section{Checkerboard Test of Antimicrobial Orbifloxacin Combinations}

Checkerboard assays with $M t b \mathrm{H} 37 \mathrm{Ra}$ were used to assess the synergic activity of orbifloxacin in combination with first-line anti-TB drugs as described in previous studies (Bonapace et al., 2002; Bhusal et al., 2005). The activity of orbifloxacin was tested in combination with RIF, INH, streptomycin, kanamycin, ethambutol, and ethionamide. A total of $100 \mu \mathrm{l}$ of the bacterial 
suspension was added to 96-well microplates as previously described. A serial twofold dilution of orbifloxacin, ranging from 1/8-fold MIC to fourfold MIC, was added to each row, and the other drugs, diluted from 1/8-fold MIC to fourfold MIC, were added to each column. Each combination was tested in triplicate for biological statistics. The plates were incubated at $37^{\circ} \mathrm{C}$ for 7 days, and bacterial growth was visually assessed. The fractional inhibitory concentration (FIC) index for each drug combination was calculated as follows:

$$
\begin{aligned}
\text { FIC index }(\mathrm{FICI})= & \text { combination } \mathrm{MIC} \mathrm{A} /{ }_{A}+ \\
& \text { combination } \mathrm{MIC} \mathrm{B} / B
\end{aligned}
$$

The combination MIC is the concentration of a single drug in the mixture that can inhibit the growth of the bacteria. A or B is the MIC of the drug when used on its own. The FIC index values were interpreted as follows: FIC index $\leq 0.5$, synergic effect; $0.5<$ FIC index $<1$, additive effect; $1<$ FIC index $<2$, indifferent effect; and FIC index $\geq 2$, antagonistic effect.

\section{Cell Line Culture and Cytotoxic Assays}

RAW264.7 (ATCC, TIB-71) cells were maintained in highglucose Dulbecco's modified Eagle's medium (DMEM, Gibco), and THP-1 cells were cultured in RPMI-1640 medium supplemented with $10 \%$ fetal bovine serum (FBS, Gibco), 50 units/ml penicillin, and $50 \mu \mathrm{g} / \mathrm{ml}$ streptomycin (Gibco) at $37^{\circ} \mathrm{C}$ in a $5 \% \mathrm{CO}_{2}$ incubator. For the cytotoxicity assay, $5 \times 10^{3}$ RAW264.7 or THP-1 cells were seeded into 96-well microplates and incubated overnight. The cells were treated with a range of concentrations (from 0 to $64 \mu \mathrm{g} / \mathrm{ml}$ ) of different drugs or peptides for $48 \mathrm{~h}$, and it also applied to the $50 \%$ effective cytotoxic concentration value determination $\left(\mathrm{CC}_{50}\right)$. Then, $10 \mu \mathrm{l}$ of the MTS kit reagent solution was added. DMSO was used as the negative control. After $4 \mathrm{~h}$ of incubation, cell viability and cytotoxicity were measured using a CellTiter $96 \mathrm{AQ}_{\text {ueous }}$ One Solution Cell Proliferation Assay (MTS, Promega, Cat\# G3580), and the absorbance was recorded at $490 \mathrm{~nm}$ using a 96-well microplate reader. All cell experiments were conducted using no more than four generations of subculture after thawing of the cell stocks. A mycoplasma-free test was performed by using the myco-blue mycoplasma detector (D101-02, Vazyme, Nanjing, China).

\section{Drug Susceptibility Testing Against Intracellular Mtb}

For in vitro antimicrobial activity studies, $2 \times 10^{5}$ RAW264.7 cells were seeded into 12 -well plates overnight and infected with $\mathrm{H} 37 \mathrm{Ra}$ at a multiplicity of infection of 5 for $4 \mathrm{~h}$ at $37^{\circ} \mathrm{C}$ under a $5 \% \mathrm{CO}_{2}$ atmosphere. Cells were washed three times with sterile PBS to stop infection. Fresh DMEM supplemented with $10 \%$ FBS, containing orbifloxacin $(4 \mu \mathrm{g} / \mathrm{ml})$, peptide $(12.8 \mu \mathrm{g} / \mathrm{ml})$, and a combination of orbifloxacin and peptide, was applied to each well. Kanamycin was used as a positive control against extracellular $M t b$. After incubation for $48 \mathrm{~h}$, the cells were washed with PBS and lysed with sterile $0.1 \%$ Tween 80 . Cell lysates were diluted and plated on 7H11 agar plates. CFUs were enumerated after 3-4 weeks of incubation.

\section{Whole-Genome Sequencing of the Drug-Resistant Mutants}

Drug resistance was induced in Mtb H37Ra by co-culturing it with orbifloxacin at a concentration of $1 / 4$-fold to 10 -fold MIC for three generations until the last generation showed a visible growth of $\mathrm{Mtb}$ in the presence of 10-fold MIC of orbifloxacin. Drug-resistant mutants were isolated from a single colony and grown on a 7H11 agar plate containing a 10fold MIC concentration of orbifloxacin. Genomic DNA was extracted using the cetyltrimethylammonium bromide-phenol chloroform method. Briefly, bacterial pellets were re-suspended in GTE lysis buffer (50 $\mathrm{mM}$ glucose, $25 \mathrm{mM}$ Tris, $10 \mathrm{mM}$ EDTA; pH 8.0) containing lysozyme at a final concentration of $100 \mu \mathrm{g} / \mathrm{ml}$ and incubated at $37^{\circ} \mathrm{C}$ overnight. RNase A $(10 \mu \mathrm{g} / \mathrm{ml})$, $2 \%$ SDS solution, and protease $\mathrm{K}(10 \mu \mathrm{g} / \mathrm{ml})$ were added, and the mixture was incubated at $56^{\circ} \mathrm{C}$ for $2 \mathrm{~h}$. Chloroformisopentanol and $75 \%$ ethanol were then used to isolate and purify the genomic DNA. The isolated DNA was quantified using a Nanodrop 2000 spectrophotometer (Thermo Fisher, United States). The DNA ( $2 \mu \mathrm{g}$ ) was fragmented by sonication and purified by gel extraction kit (Cat\# D2500-02, OMEGA Bio-tek, United States). Fragmented DNA was repaired using the Hieff NGS Fast-Pace End Repair/dA-Tailing Module (Cat\# 12608, YEASEN, United Kingdom) and ligated with a Y-adapter. Paired-end DNA libraries were constructed using a 14-cycle PCR amplification for Illumina next-generation sequencing (Hiseq 2000 platform). The quality of the DNA library was measured using Qubit 4 fluorometer (Invitrogen), and the DNA library was sent to Annoroad Gene Technology Company (Beijing) for high-throughput DNA sequencing.

\section{Determination of the Structure of the Ligand-Receptor Complex by Molecular Docking}

The structure of orbifloxacin as a complex with $M t b$ GyrA was determined using Discovery Studio 2018 software based on the receptor-ligand interaction properties. The X-ray crystal structure of $M t b$ GyrA (PDB code: 5BS8) was obtained from the RCSB Protein Data Bank ${ }^{1}$. The 3D structure of orbifloxacin (PubChem ID: 60605) was downloaded from the open chemistry database PubChem ${ }^{2}$. The amino acid residue Asp94 of the wildtype protein was replaced with Gly94 to create a mutant GyrA crystal structure, using the Build and Edit Protein module of the software. The drug-protein binding model was predicted using the CDOCKER docking module, based on receptorligand interaction properties. The water molecules in the original crystal structure were removed prior to molecular docking. The binding site of the protein and orbifloxacin was found to be the active site cavity of GyrA, which is composed of A:Ala90, A:Asp94/Gly94, B:Gly459, B:Arg482, B:Thr500; B:Glu501, C:Arg128, and C:Ptr129. The default settings of CDOCKER were used. Docking results were analyzed and

\footnotetext{
${ }^{1}$ https://www.rcsb.org

${ }^{2}$ https://pubchem.ncbi.nlm.nih.gov
} 
visualized using the "View Interactions" module in receptorligand interaction properties.

\section{Cloning of the Point Mutant and Wild-Type gyrA (Rv0006) Gene}

Primers were designed based on sequences obtained from the National Center for Biotechnology Information (NCBI) ${ }^{3}$. The full-length of gyrA gene (Rv0006) amplified from the orbifloxacin-resistant strain and the wild-type strain was inserted into the plasmid, pMV261-GroEL, to obtain pMV261-GroELMutant gyrA and pMV261-GroEL-WT gyrA, respectively. The recombinant plasmids were electroporated into Mtb H37Ra competent cells using an electric pulse machine for $20 \mathrm{~ms}$, under a running program of $2.5 \mathrm{kV}, 1,000 \Omega$, and $25 \mu \mathrm{F}$, and then plated onto Middlebrook $7 \mathrm{H} 11$ agar plates containing kanamycin $(100 \mu \mathrm{g} / \mathrm{ml}$ ) or orbifloxacin (five-fold MIC, $1 \mu \mathrm{g} / \mathrm{ml}$ ). Single colonies were grown for MIC determination after 4 weeks of culture containing the antibiotic selection.

\section{Animal Experiments}

C57BL/6 mice (female mice, 6-8 weeks old, $20 \pm 2$ g) were purchased from Beijing Vital River Laboratory Animal Technology and raised under specific pathogen-free conditions at the Laboratory Animal Center of Huazhong Agricultural University. All animals were randomly divided into three groups of 6-8 mice in each. Mice were infected intravenously with $0.2 \mathrm{ml} \mathrm{M} t b \mathrm{H} 37 \mathrm{Ra}$ suspension at a dose of $5 \times 10^{6} \mathrm{CFU} / \mathrm{ml}$ for 4 days. The infected mice were then orally administered orbifloxacin at a dose of $50 \mathrm{mg} / \mathrm{kg}$ per day. Isoniazid was used as a positive control at a dose of $12.5 \mathrm{mg} / \mathrm{kg}$, and PBS was used as a negative control. The drugs were administered to the mice for 7 days post of infection. The mice were euthanized 18 days after infection. The lungs were used for histopathological and CFU analysis. A part of the left lung was fixed in $4 \%$ paraformaldehyde for $48 \mathrm{~h}$, and tissues were embedded in paraffin to prepare sections for hematoxylin and eosin staining. Homogenates of lungs were plated onto Middlebrook 7H11 agar, containing OADC enrichment and BBL MGIT PENTA antibiotics (Cat\# 245114, BD, United States), for bacterial burden enumeration. CFUs were counted after $3-4$ weeks of incubation at $37^{\circ} \mathrm{C}$.

\section{Cytokine Analysis}

RNA was extracted from the lungs of mice, using the chloroform-isopropanol method. RNA was reverse transcribed using ReverTra Ace qPCR RT Master Mix, with a gDNA Remover Kit (Code No. FSQ-301, TOYOBO, Japan), according to the manufacturer's instructions. SYBR Select Master Mix (Cat\# 4472919, Thermo Fisher, United States) was used for the detection of murine tumor necrosis factor $\alpha$ (TNF- $\alpha$ ), interleukin (IL) 4, IL-1 $\beta$, IL-6, IL-10, and interferon $\beta$ (IFN- $\beta$ ), and glyceraldehyde 3-phosphate dehydrogenase (GAPDH) was used as the housekeeping gene. Real-time PCR was run on a QuantStudio 6 real-time PCR system (ABI, United States), and fold change expression was calculated using the $2^{-\Delta \Delta C t}$ method normalized to GAPDH (Emily et al., 2021).

\footnotetext{
${ }^{3}$ https://www.ncbi.nlm.nih.gov/gene
}

\section{Data Availability}

The raw data of the whole-genome sequence was submitted to the Sequence Read Archive (SRA) on the NCBI website (BioProject accession number: PRJNA661429).

\section{Statistical Analysis}

Numerical data from three independent experiments were analyzed using GraphPad Prism 6.0 (La Jolla, CA, United States) and are shown as mean \pm standard deviation or standard error of the mean. The significance of differences between the groups was evaluated using one-way ANOVA or Student's $t$-test. Statistical differences were considered to be significant at $p<0.05$; $p$ values of $<0.05,<0.01,<0.001$, and $<0.0001$ are indicated as *,**, ***, and ${ }^{* * *}$, respectively.

\section{RESULTS}

\section{Screening the Small Molecule Library Against Mtb}

A library of compounds, including 502 small molecules of natural origin and their derivatives, 299 and 269 bioactive compounds isolated from traditional Chinese medicine recipes, seven small peptides, and 640 FDA-approved drugs were screened for their anti-TB activity, based on their effect on the growth of $M t b$ in vitro (Figures $\mathbf{1 A}, \mathbf{B}$ ). The large-scale screening of the compounds in microplates (Supplementary Figure 1A) showed that antibiotics including orbifloxacin, prulifloxacin, nadifloxacin, disulfiram, and mithramycin, and antimicrobial peptides including AK15, AK15-7COOH, LZ1, and ZY4 exhibited significant anti- $M t b$ activity (Figure 1C). Amongst the compounds tested, the fluoroquinolones (orbifloxacin, prulifloxacin, and nadifloxacin) and mithramycin were found to show the best anti-TB activity, exhibiting MICs of $0.2-0.4 \mu \mathrm{g} / \mathrm{ml}$. The peptide AK15 also presented favorable activity against $M t b$ with a MIC of $128 \mu \mathrm{g} / \mathrm{ml}$, and the remaining peptide (AK15$7 \mathrm{COOH}, \mathrm{LZ1}$, and ZY4) showed activity against Mtb with MIC of $256 \mu \mathrm{g} / \mathrm{ml}$ (Figure 1D). These data indicated that the drugs and peptides could have a potential antimicrobial activity on $M t b$. The structures of the two bioactive peptides, AK15 and $15 \mathrm{AK}-7 \mathrm{COOH}$, are shown in Supplementary Figure 1B. The chemical structures of the small molecules were obtained from the PubChem database (see text footnote 2) and shown in Figure 1E.

\section{Intracellular Bactericidal Effect of Orbifloxacin}

The cytotoxicity of the selected antibiotics (orbifloxacin, prulifloxacin, nadifloxacin, and mithramycin) and peptides (AK15, AK15-7COOH, LZ1, and ZY4) was tested using the MTS method. A decrease in $\mathrm{OD}_{490 \mathrm{~nm}}$ indicated cytotoxicity toward the RAW264.7 cell. We found that orbifloxacin and prulifloxacin were not cytotoxic at a concentration of $8 \mu \mathrm{g} / \mathrm{ml}$, whereas nadifloxacin and mithramycin were cytotoxic at $16 \mu \mathrm{g} / \mathrm{ml}$ to RAW264.7 cells (Figure 2A), but showed distinct cytotoxicity toward THP-1 cells even at low concentrations 
A Information of compound libraries

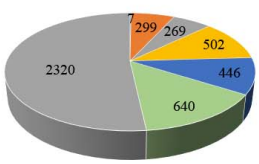

- Peptides

- CYX library

- LXD library

- Natural products library

- NIH clinical library

- FDA-approved library

The spectrum library

C
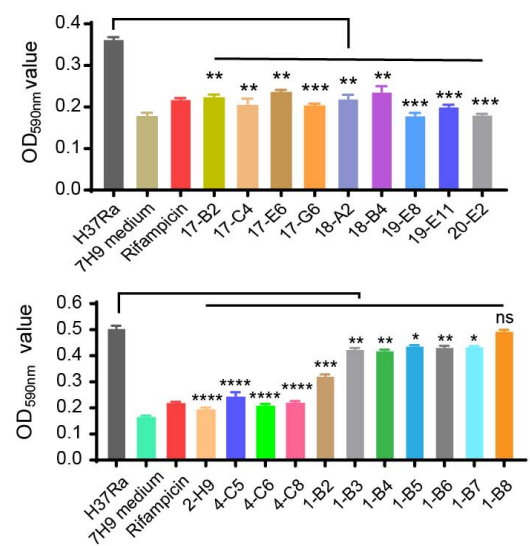

E<smiles>CC1CN(c2c(F)c(F)c3c(=O)c(C(=O)O)cn(C4CC4)c3c2F)CC(C)N1</smiles>

Orbifloxacin (113617-63-3)

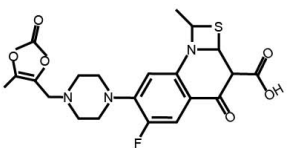

Prulifloxacin (123447-62-1)
B

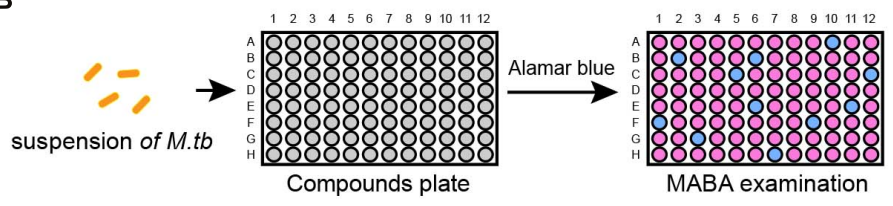

D

- ethacridine lactate

$=$ nitroxoline

= orbifloxacin

= homidium bromide

- acriflavinium

= prulifloxacin

alexidine

nadifloxacin

bleomycin sulfate

- pleomycin su

= mitomycin c

- AK15

= AK15-7 $\mathrm{COOH}$

피 $\mathrm{Z} 4$

- $\mathrm{LZ1}$

$=\mathrm{FL}-12$

\begin{tabular}{lcccl}
\hline Compounds & Solvent & $\mathrm{MIC}(\mu \mathrm{g} / \mathrm{mL})$ & Con. $(\mu \mathrm{M})$ & Bioactivity \\
\hline Orbifloxacin & $0.5 \%$ Acetic acid & 0.2 & 0.505 & antibacterial \\
Prulifloxacin & $0.5 \%$ Acetic acid & 0.4 & 0.867 & antibacterial \\
Nadifloxacin & DMSO & 0.2 & 0.555 & antibacterial \\
Mithramycin & PBS & 0.4 & 0.369 & antineoplastic \\
AK15 & Sterilized ddH2O & 128 & 70.1 & unknown \\
AK15-7-COOH & Sterilized ddH2O & 256 & 140.1 & unknown \\
LZ1 & Sterilized ddH2O & 256 & N.D & unknown \\
ZY4 & Sterilized dd H2O & 256 & N.D & unknown \\
IN-14 & PBS & 256 & N.D & unknown \\
FL-12 & PBS & 256 & N.D & unknown \\
GQ-28 & PBS & 512 & N.D & unknown \\
RIF & DMSO & 0.025 & 0.03 & antitubercular \\
\hline
\end{tabular}

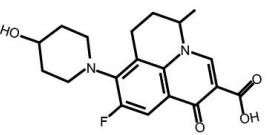

Nadifloxacin (124858-35-1)

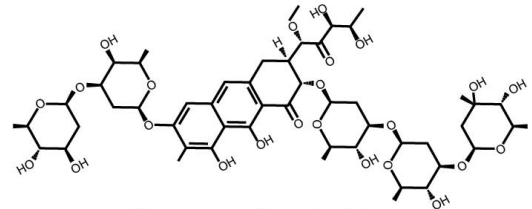

Mithramycin (18378-89-7)

FIGURE 1 | In vitro screening of drug libraries against Mycobacterium tuberculosis H37Ra. (A) The distribution of each group of compounds in the drug libraries. (B) Procedure for the drug screening: a defined concentration of each compound from the libraries was inoculated with a suspension of $M$ tb in 96 -well microplates for 7 days, and the antibacterial effects were determined by Alamar Blue assay. (C) Graphical representation of the antibacterial effects of selected compounds. (D) Determination of MIC of selected compounds and peptides, as described in the chart. Rifampicin (RIF) was used as a positive control. These results are presented as mean \pm SD from triplicates. Two-tailed unpaired Student's $t$-test was used for analysis: N.D., not detectable; ${ }^{\star} p<0.05$; ${ }^{\star \star} p<0.01$; ${ }^{\star \star \star} p<0.001$; ${ }^{\star \star \star \star} p<0.0001$. (E) Chemical structures of orbifloxacin, prulifloxacin, nadifloxacin, and mithramycin.

(Supplementary Figure 2A). Orbifloxacin also displayed a very high safety cytotoxic concentration to Raw264.7 cell with $115.42 \mu \mathrm{M}(\mathrm{MIC}=0.51 \mu \mathrm{M})$ (Supplementary Figure 3). The peptides AK15, AK15-7COOH, and ZY4 did not show any cytotoxicity up to $64 \mu \mathrm{g} / \mathrm{ml}$; the maximum safe concentration of LZ1 was found to be $16 \mu \mathrm{g} / \mathrm{ml}$ (Figure 2B).

Next, we examined the effects of these drugs on the clearance of intracellular $M t b$ in macrophages by CFU assay (Figure 2C). We found that the number of CFUs from orbifloxacin-treated cells was significantly lower than that in the DMSO-treated control. Notably, there were no significant differences between the CFUs observed in the orbifloxacin compared with the isoniazid group. A significant difference was observed between the orbifloxacin and kanamycin (control for extracellular $M t b$ inhibition) groups. A combination of orbifloxacin with INH produced a significant decrease in the number of CFUs compared with treatment with either drug alone (Figure 2D). The peptides AK15, AK15-7COOH, LZ1, and ZY4 did not affect the clearance of intracellular $M t b$, despite their in vitro efficacy (Figure 2C). Interestingly, a combination of these peptides with orbifloxacin significantly enhanced the effects of orbifloxacin in clearing intracellular Mtb (Figure 2D). However, a similar effect was not observed when they were used in combination with prulifloxacin (Figure 2E). Furthermore, we evaluated the activity of orbifloxacin in combination with first-line antibiotics (rifampicin, isoniazid, and ethambutol) and found that a combination of these drugs has four times more potent bactericidal activity than any of the drugs alone (Supplementary Figure 2B).

\section{In vivo Activity of Orbifloxacin}

To further validate the anti-Mtb effect of orbifloxacin in vivo, we infected C57BL/6 mice with $M t b \mathrm{H} 37 \mathrm{Ra}$ and administered the drugs 4 days after infection for a total of 7 days. The mice were then sacrificed on day 18 postinfection for histopathological and bacterial burden analysis (Figure 3A). The mice in the orbifloxacin and INH groups (isoniazid used as a drug positive control) showed a slight weight loss (Figure 3B), with a significant reduction in the number of CFUs relative to that in the PBS control. There was no significant difference between the groups treated with orbifloxacin and INH (Figure 3B). Histopathological examination of lung tissues revealed that PBS-treated mice showed moderately higher infiltration of inflammatory cells, such as macrophages and lymphocytes, than mice treated with the drugs (Figure 3C). In PBS-treated mice, thickening of the alveolar wall, narrowing of the alveolar space, 
A

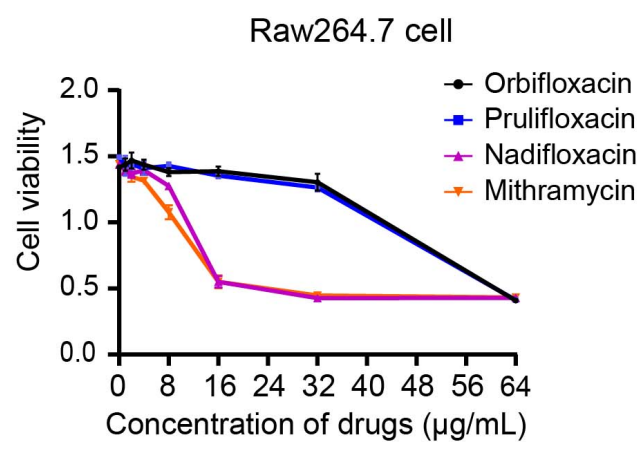

B

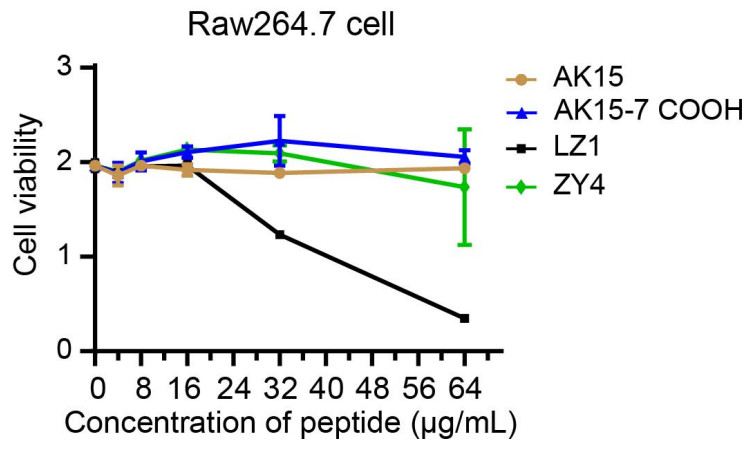

C

C Raw264.7 cell

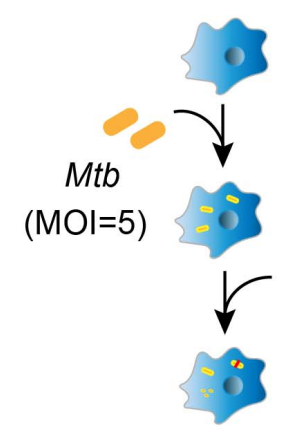

tested

compounds

(48h)

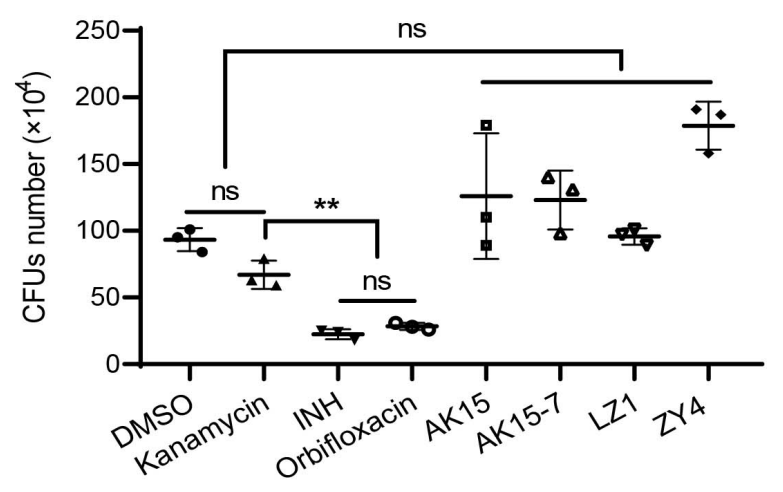

D

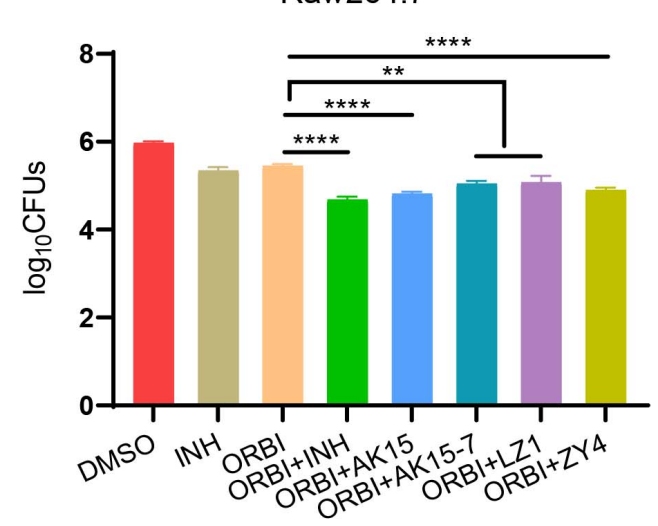

$48 \mathrm{hr}$ post of infection (MOI=5)
E

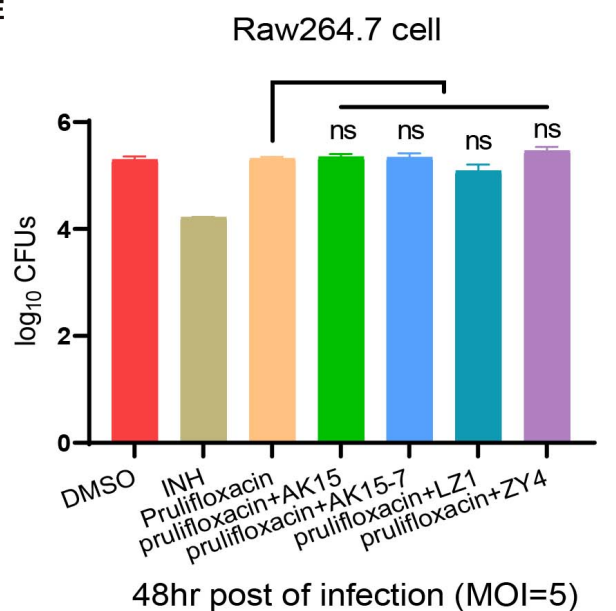

FIGURE 2 | Cytotoxicity detection and intracellular anti-Mycobacterium tuberculosis activities of selected drugs. (A,B) A cell proliferation assay was used to detect the toxicity of orbifloxacin, prulifloxacin, nadifloxacin, mithramycin, and the peptides on RAW264.7 macrophages. (C) Procedure for intracellular drug susceptibility test in RAW264.7 cells and CFU enumeration of Mtb in murine macrophages treated with individual drugs for $48 \mathrm{~h}$ post infection. (D) Combination effects of orbifloxacin with the peptides against Mtb in vitro. (E) Combination effects of prulifloxacin with the peptides against Mtb in vitro. These experiments were performed in duplicate and analyzed using multiple comparison test in GraphPad Prism: ns, not significant; ${ }^{\star *} p<0.01$; ${ }^{\star * \star *} p<0.0001$ (mean \pm SD from triplicates).

and the buildup of some red blood cells in the cavity were observed in the lungs. In contrast, the lesions were much milder and fewer macrophages were present in the alveolar cavity of the mice treated with orbifloxacin (Figure 3C). We also analyzed cytokine expression levels in the lungs of the mice. IL- 6 and IL-1 $\beta$ expression were significantly lower in the orbifloxacin-treated group than in the PBS-treated group (Figures 3F,H). There were no significant differences in the expression of TNF- $\alpha$ in any of the groups (Figure 3D). The expression of IL-4, the representative cytokine of Th2 response showed slightly decrease in orbifloxacin treated mice (Figure 3E). IL-10 and IFN- $\beta$ expression were significantly higher 

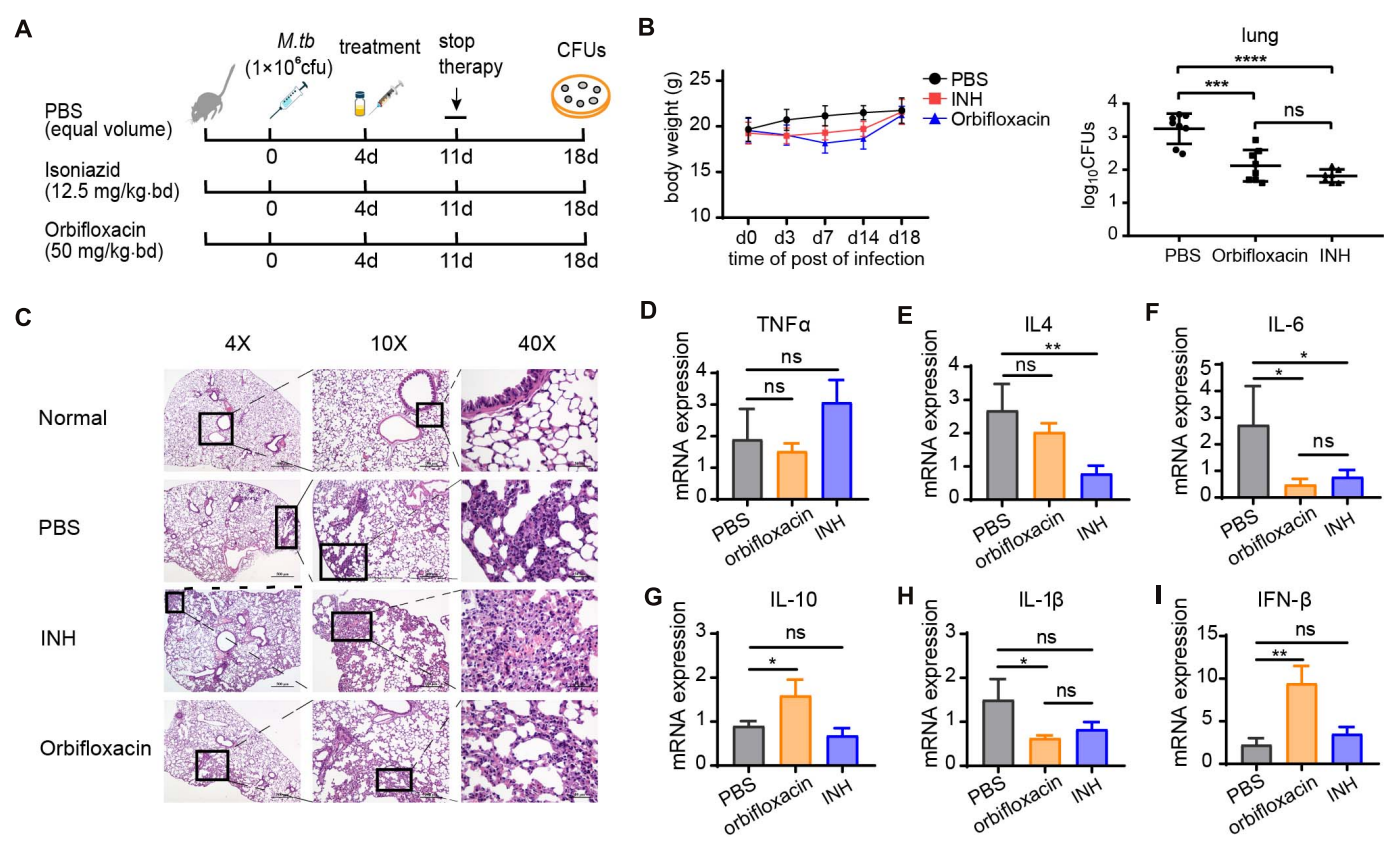

FIGURE 3 | In vivo anti-Mycobacterium tuberculosis effects of orbifloxacin in a mouse model. (A) Schematic representation of the procedure for the in vivo assay in C57BL/6 mice ( $n=7$ or 8 in each group). (B) Changes in body weight were observed, and the bacterial burden in the lungs of the infected mice was determined by plating the organ lysate on the $7 \mathrm{H} 11$ plates after treatment with orbifloxacin or PBS for 7 days. Data are presented as mean \pm SD. (C) Hematoxylin and eosin staining of lung sections of representative Mtb-infected mice from the drug-treated group and control group. (D-I) The expression of various cytokine genes was determined by quantitative PCR from the whole lung homogenates of mice from the drug-treated and control groups. ns, not significant; ${ }^{\star} p<0.05$; ${ }^{\star \star} p<0.01$; ${ }^{\star * *} p<0.001 ;{ }^{* \star * *} p<0.0001$.

in the orbifloxacin-treated group than in the INH- or PBStreated groups (Figures 3G,I), suggesting that orbifloxacin can protect mice from a strong inflammatory response. Overall, our results show that orbifloxacin exhibits strong in vivo anti-Mtb activity and could be considered as a potential drug for the treatment of TB.

\section{GyrA D94G Mutation Is Attributed to Orbifloxacin Resistance in Mtb}

To understand the cause of orbifloxacin resistance in $M t b$, we induced the generation of orbifloxacin-resistant in an $M t b$ strain and confirmed the resistance using a MIC assay (Figure 4A). Because combination therapy using orbifloxacin and INH enhances the bactericidal action, we aimed to detect any potential cross-resistance to INH by culturing orbifloxacin-resistant strains of $M t b$ in the presence of INH. These mutated strains did not exhibit resistance to INH (Figure 4B). Next, five colonies of orbifloxacin-resistant $\mathrm{Mtb}$ were subjected to whole-genome sequencing, and 17 single nucleotide point mutations were observed, compared with the wild-type strain ATCC25177 (Figure 4C, circular map). Notably, mutations in gyrA were observed in all five resistant strains. Thus, we hypothesized that the gyrA gene might be the key to understanding orbifloxacin resistance on $M t b$. Therefore, we analyzed the gene sequence of gyrA and found a single point mutation at the 94th amino acid residue, where the aspartic acid was replaced by glycine (GyrA D94G) (Figure 4D).

To confirm whether this point mutation is responsible for orbifloxacin resistance, we introduced a point mutation of D94 to G94 in the gyrA gene into wild-type $M t b$ as above. We found that the mutant $M t b$ strain could grow on a Middlebrook 7H11 agar plate supplemented with a fivefold MIC concentration of orbifloxacin $(1 \mu \mathrm{g} / \mathrm{ml})$, whereas $M t b$ transfected with a plasmid control or with a wild-type gyrA gene could not (Figure $4 \mathrm{E}$ ). To further confirm the resistance, we grew a single colony of $M t b$ containing the mutation in $g y r A$ in a 96 -well plate in the presence of orbifloxacin and found that the MIC increased from 0.2 to $3.2 \mu \mathrm{g} / \mathrm{ml}$, which displayed a stronger resistance activity to orbifloxacin (Figure 4F). These data suggest that the gyrA gene of $M t b$ is the target of orbifloxacin and that the gyrA D94G mutation may be responsible for resistance to orbifloxacin.

\section{Analysis of Ligand-Receptor Interactions by Structural Docking}

To explore the molecular mechanism of action of orbifloxacin through its action on $g y r A$, we first modeled the crystal structure of the mutant gyrase subunit A, using Discovery Studio 2018 (Figure 5B), based on the structure of the wild-type $M t b$ gyrase (Figure 5A). Subsequently, the binding patterns of orbifloxacin with wild-type and mutant gyrase were modeled using the CDOCKER docking module, based on the receptor-ligand 
A

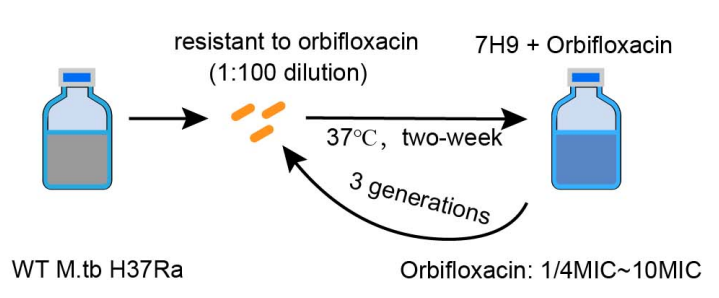

B

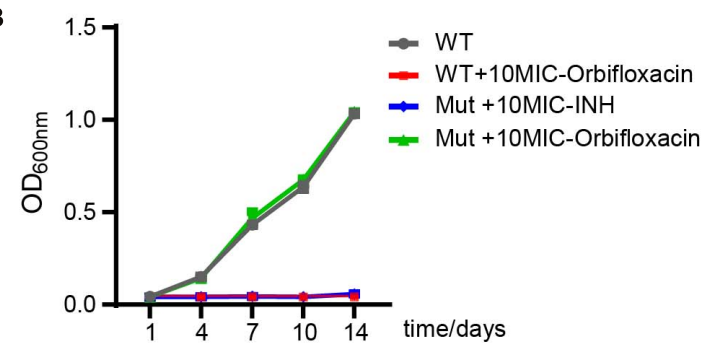

C

D

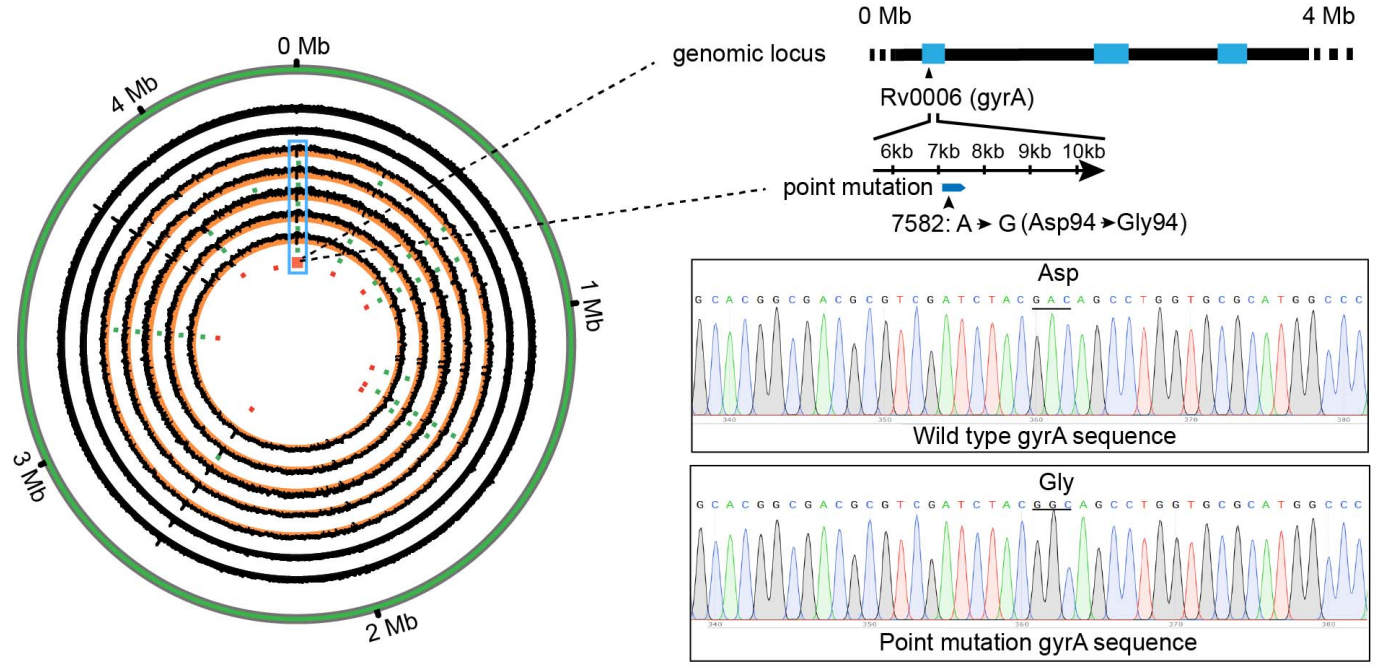

E

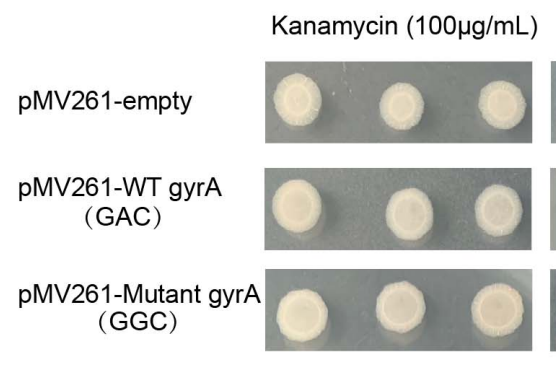

$\mathbf{F}$

F Orbifloxacin concentration $(\mu \mathrm{g} / \mathrm{mL})$
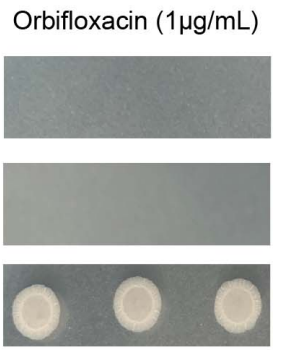

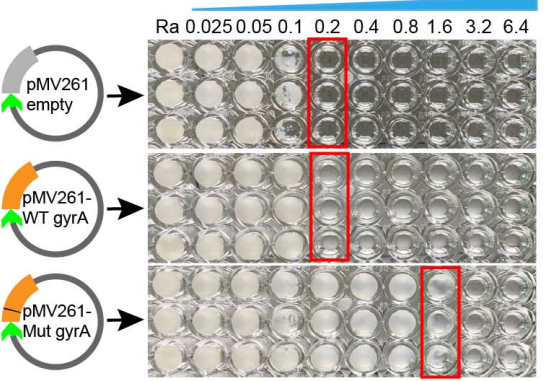

FIGURE 4 | Whole-genome sequencing analysis of the orbifloxacin-resistant strain. (A) Wild-type Mtb H37Ra was treated with orbifloxacin at concentrations of $1 / 4$-fold MIC to 10 -fold MIC to induce drug resistance. (B) The acquired orbifloxacin resistance of the mutated strain was confirmed by comparing its growth on $7 \mathrm{H} 9$ medium, supplemented with 10-fold MIC of orbifloxacin, with that of the wild-type strain. (C) Circos plot represents the whole-genome sequence of the wild-type strain and the five orbifloxacin-resistant strains. (D) The point mutation of GAC to GGC in the Rv0006 gene (gyrA) is displayed as confirmed by Sanger sequencing. (E) Over-expression of the wild-type gyrA and mutant gyrA gene in wild-type $M$ tb and the growth of bacteria in the presence of $1 \mu \mathrm{g} / \mathrm{ml}$ of orbifloxacin.

pMV261-empty and pMV261-WT gyrA were used as controls. (F) MIC of orbifloxacin in the Mtb strain expressing mutant gyrA [obtained from the plates in panel (D)]

interaction properties. Orbifloxacin was seen to occupy the active site of the wild-type protein GyrA (Figure 5C). Orbifloxacin binding was found to be controlled by the Asp94, Arg128, Ptr129, Gly483, Glu459, and Asp532 residues in the binding site (Figure 5D). Ptr129 and Glu459 played an important role in this binding, forming hydrogen bonds with orbifloxacin. A favorable $\pi$-cation interaction between the benzene ring of orbifloxacin and the backbone hydrogen nitride of Arg128 was also predicted by the software. In addition, a single active site magnesium ion bridged the hydroxyl oxygen of Asp94 with the fourth and sixth oxygens of orbifloxacin. However, due to the difference between the side chains of Asp and Gly (Figures 5E,F), the corresponding favorable interaction was disrupted in the mutant protein GyrA, the details of which are depicted in Figures 5G,H. Our data suggest that D94G impairs the binding of orbifloxacin with protein GyrA, which could explain the mechanism of resistance to orbifloxacin in $M t b$. 
A

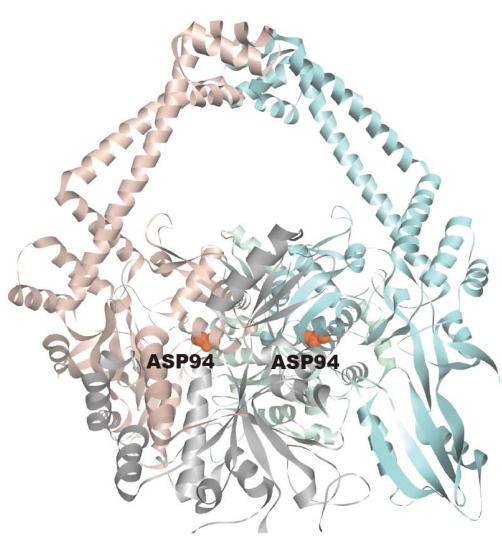

C

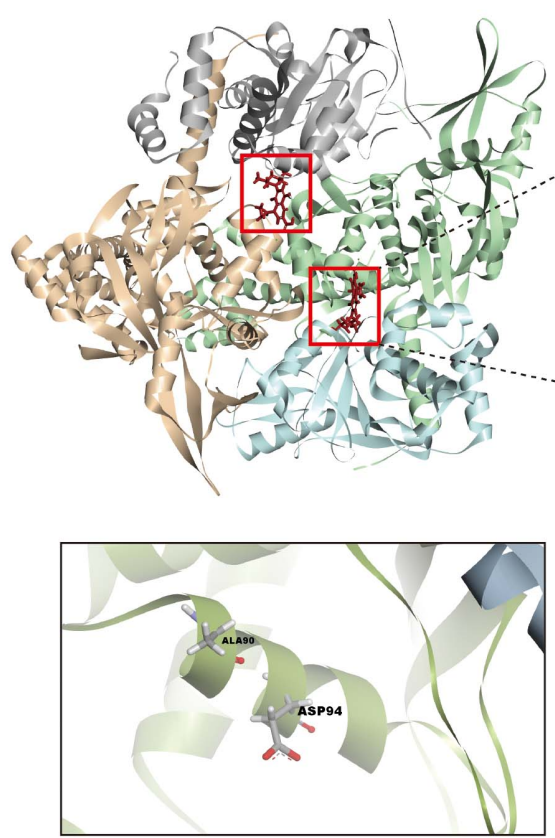

F

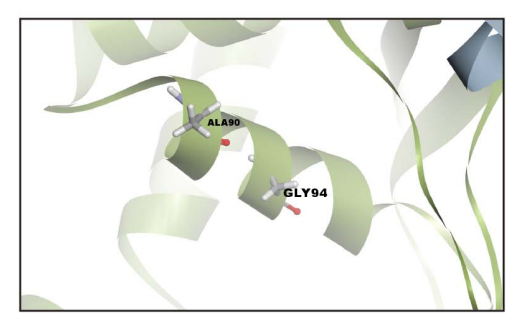

B

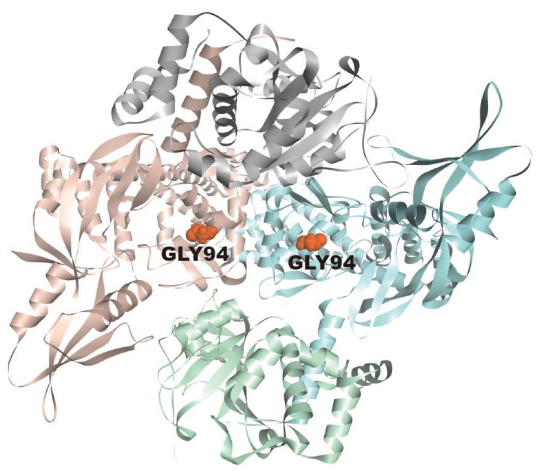

D

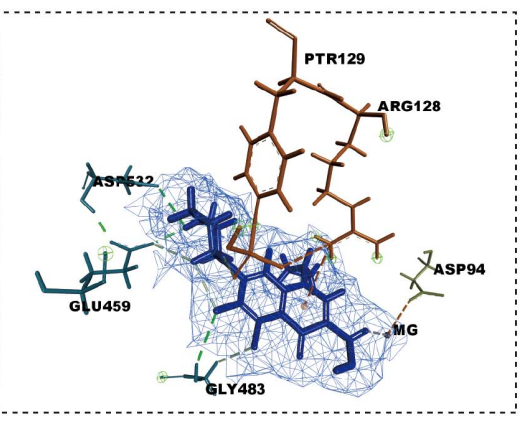

G

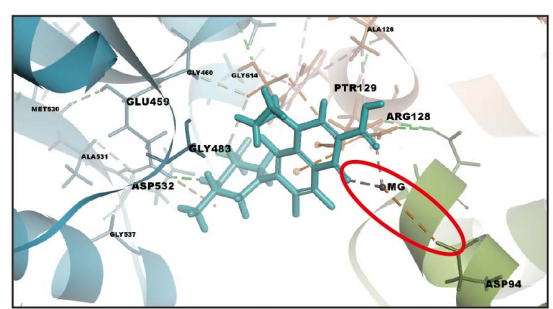

H

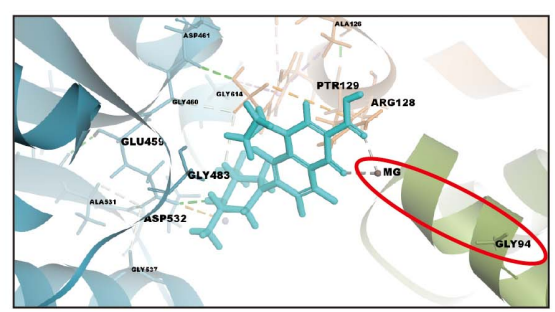

FIGURE 5 | Structural analysis of the GyrA-orbifloxacin complex and comparison of the binding of orbifloxacin to GyrA wild-type and GyrA D94G. (A,B) Overall structure of wild-type (A) and remodeled mutant gyrA protein (B). gyrA ${ }^{D 94 G}$ was created by replacing the 94th amino acid residue, Asp, with Gly in GyrA wild-type. (C) A cartoon representation of the GyrA wild-type-orbifloxacin complex. Orbifloxacin binding is mainly controlled by residues in the active site cavity of GyrA wild-type. (D) The detailed interactions and key contact sites of the complex. The residues that interact with orbifloxacin are marked. (E,F) Amino acid changes in GyrA D94G compared with GyrA wild-type. The side chains of the 94th amino acid residue in GyrA wild-type and GyrA D94G are labeled. (G,H) Comparison of binding interactions between GyrA wild-type-orbifloxacin and GyrA D94G-orbifloxacin. Magnesium ion-bridged interaction between the hydroxyl oxygen of Asp94 in gyrA with the fourth and sixth oxygen of orbifloxacin. This interaction in wild-type gyrA is disrupted by the D94G mutation.

\section{DISCUSSION}

Tuberculosis is a white plague that kills millions of people every year. Several drugs have been developed and used clinically for TB for decades (Yuan and Sampson, 2018). Conventional drugs take more than half a year to effectively treat the infection; however, many patients are unable to complete the course of treatment due to its serious side effects. In addition, long-term use of 
these antibiotics has led to the emergence of drug resistance, which poses a huge threat to human life. MDR and XDR Mtb infections have been reported worldwide (Seung et al., 2015; Sander et al., 2020). In this study, we found that orbifloxacin had high anti-Mtb bioactivity in vitro with a low MIC of $0.2 \mu \mathrm{g} / \mathrm{ml}$ and protected the mice from infection in vivo. Interestingly, the use of orbifloxacin in combination with INH and RIF exhibited more potent bactericidal activity against Mtb in vitro. Such combination therapy not only reduces the required dose and the frequency of drug administration but also reduces the duration of treatment, thus reducing side effects and the risk of developing drug resistance. Therefore, combination therapy involving orbifloxacin could be further evaluated as a new treatment regimen for TB.

Antibiotics without cross-resistance, as well as combination therapies, have been assessed in clinical trials for the treatment of patients with INH-resistant TB (Hu et al., 2017; Tweed et al., 2019). In the current study, we found that a mutation of the 94th amino acid of protein GyrA is likely to be responsible for resistance to orbifloxacin, without affecting INH susceptibility because of the different target on the mechanism of action such as katG gene or inhA (Arash et al., 2019; Flentie et al., 2019). Although mutations in the gyrA gene have been linked to fluoroquinolone resistance in $M t b$ such as moxifloxacin (Aldred et al., 2016; Blower et al., 2016; Petrella et al., 2019), the detailed mechanism of $M t b$ resistance to orbifloxacin has not been elucidated. With the low cure rate and high recurrence rate of drug-resistant $\mathrm{TB}$, the discovery of orbifloxacin targets and elucidation of mechanisms of orbifloxacin resistance facilitate effective therapy for no cross drug-resistant TB and may offer a good basis for the rational design of treatment of drug-resistant TB. Data from computational studies in Figure $\mathbf{5}$ demonstrate the interactions of $M t b$ with orbifloxacin via a magnesium bridge from the Asp94 in the active site. This interaction was also predicted to be the mechanism underlying fluoroquinolone resistance (Yu et al., 2017; Rhastin et al., 2020). Understanding this mechanism provides a promising strategy for the study and development of synthetic drugs using orbifloxacin and its derivatives to face drug resistance challenges.

In this study, we screened a library of small molecules and peptides and identified orbifloxacin as a potential drug candidate for anti-TB treatment. No cross-resistance was observed between isoniazid and orbifloxacin because of the different targets; this drug might be useful for the treatment or combination therapy of INH-resistant TB. Moreover, using an orbifloxacinGyrA enzyme model, we found that a single mutation in gyrA (Gyrase subunit A D94G) is able to reduce the interaction of orbifloxacin with protein GyrA. These data can enable the development of orbifloxacin derivatives that can bind to this mutant site for high efficiency against drug-resistant $M t b$ and thus be used in the treatment of fluoroquinoloneresistant $M t b$. In conclusion, our study identified a potential anti-TB drug candidate and elucidated its mechanism of action, which could contribute to the rational design of more potent anti-TB drugs and help combat MDR and XDR-TB.

\section{DATA AVAILABILITY STATEMENT}

The datasets presented in this study can be found in online repositories. The names of the repository/repositories and accession number(s) can be found below: https://www.ncbi.nlm. nih.gov/, PRJNA661429.

\section{ETHICS STATEMENT}

The animal study was reviewed and approved by the committee on the ethics of animal experiments of the college of veterinary medicine, Huazhong Agricultural University (HZAUMO-2019038).

\section{AUTHOR CONTRIBUTIONS}

WZ, BY, YZ, KR, XC, and GC: data curation. ZF, XC, and GC: funding acquisition. WZ and BY: investigation and writing original draft. WZ, BY, and GC: project administration. RL: resources. $\mathrm{ZF}$ and GC: supervision. WZ: validation. $\mathrm{YZ}, \mathrm{KR}$, XJC, YL, XC, and GC: writing review and editing. All authors contributed to the article and approved the submitted version.

\section{FUNDING}

This work was supported by the National Key Research and Development Program of China under Grant No. 2017YFD0500303; National Natural Science Foundation of China under Grant Nos. 31602061, 31872470, and 31902240; the China Postdoctoral Science Foundation under Grant No. 2018M640718; and the Natural Science Foundation for Youths of Hubei Province of China, under Grant No. 2019CFB126.

\section{ACKNOWLEDGMENTS}

We would like to thank the State Key Laboratory of Agricultural Microbiology and Laboratory Animal Center of Huazhong Agricultural University.

\section{SUPPLEMENTARY MATERIAL}

The Supplementary Material for this article can be found online at: https://www.frontiersin.org/articles/10.3389/fmicb. 2021.658637/full\#supplementary-material

Supplementary Table 1 | Primers used for qRT-PCR. 


\section{REFERENCES}

Adams, K. N., Takaki, K., Connolly, L. E., Wiedenhoft, H., Winglee, K., Humbert, O., et al. (2011). Drug tolerance in replicating mycobacteria mediated by a macrophage-induced efflux mechanism. Cell 145, 39-53. doi: 10.1016/j.cell. 2011.02.022

Aggarwal, A., Parai, M. K., Shetty, N., Wallis, D., Woolhiser, L., Hastings, C., et al. (2017). Development of a novel lead that targets M. tuberculosis polyketide synthase 13. Cell 170, 249-259. doi: 10.1016/j.cell.2017.0k6.025

Akos, S., Vera, B., Rico, H., Guido, V. B., and Erik, C. B. (2015). A mutation associated with clofazimine and bedaquiline cross-resistance in MDR-TB following bedaquiline treatment. Eur. Respir. J. 45, 554-557. doi: 10.1183/ 09031936.00142914

Aldred, K. J., Blower, T. R., Kerns, R. J., Berger, J. M., and Osheroff, N. (2016). Fluoroquinolone interactions with Mycobacterium tuberculosis gyrase: enhancing drug activity against wild-type and resistant gyrase. Proc. Natl. Acad. Sci. U.S.A. 113, E839-E846. doi: 10.1073/pnas.1525055113

Arash, G., Elisa, T., Eranga, K., Stefan, N., Jennifer, P., Claudio, U. K., et al. (2019). Isoniazid resistance in Mycobacterium tuberculosis is a heterogeneous phenotype composed of overlapping MIC distributions with different underlying resistance mechanisms. Antimicrob. Agents Chemother. 63:e0009219. doi: 10.1128/AAC.00092-19

Arthur, P. K., Amarh, V., Cramer, P., Arkaifie, G. B., Blessie, E. J. S., Fuseini, M. S., et al. (2019). Characterization of two new multidrug-resistant strains of Mycobacterium smegmatis: tools for routine in vitro screening of novel anti-mycobacterial agents. Antibiotics 8:4. doi: 10.3390/antibiotics 801 0004

Bhusal, Y., Shiohira, C. M., and Yamane, N. (2005). Determination of in vitro synergy when three antimicrobial agents are combined against Mycobacterium tuberculosis. Int. J. Antimicrob. Agents 26, 292-297. doi: 10.1016/j.ijantimicag. 2005.05.005

Blower, T. R., Williamson, B. H., Kerns, R. J., and Berger, J. M. (2016). Crystal structure and stability of gyrase-fluoroquinolone cleaved complexes from Mycobacterium tuberculosis. Proc. Natl. Acad. Sci. U.S.A. 113, 1706-1713. doi: $10.1073 /$ pnas. 1525047113

Bonapace, C. R., Bosso, J. A., Friedrich, L. V., and White, R. L. (2002). Comparison of methods of interpretation of checkerboard synergy testing. Diagn. Microbiol. Infect. Dis. 44, 363-366. doi: 10.1016/S0732-8893(02)00473-X

Castro, R. A. D., Sonia, B., and Sebastien, G. (2020). The within-host evolution of antimicrobial resistance in Mycobacterium tuberculosis. FEMS Microbiol. Rev. 71, 1-27. doi: 10.1093/femsre/fuaa071

Correia, S., Poeta, P., Hebraud, M., Capelo, J. L., and Igrejas, G. (2017). Mechanisms of quinolone action and resistance: where do we stand? J. Med. Microbiol. 66, 551-559. doi: 10.1099/jmm.0.000475

Emily, D. C., Ursula, M., João, S. C. F. (2021). BMP-6 and SMAD4 gene expression are altered in the cumulus cells from women with endometriosis-associated infertility. Acta Obstet. Gynecol. Scand. 100, 868-875. doi: 10.1111/aogs.13931

Fatma, K., Laure, F. L. R., Aurélie, C., Vincent, J., Nacer, L., Koen, A., et al. (2020). Fully weekly antituberculosis regimen: a proof-of-concept study. Eur. Respir. J. 56:1902502. doi: 10.1183/13993003.02502-2019

Flentie, K., Harrison, G. A., Tükenmez, H., Livny, J., Good, J. A. D., Sarkar, S., et al. (2019). Chemical disarming of isoniazid resistance in Mycobacterium tuberculosis. Proc. Natl. Acad. Sci. U.S.A. 116, 10510-10517. doi: 10.1073/pnas. 1818009116

Fuad, M., Kerri, V., Nguyen, N. L., Licé, G. A., Medea, G., Ernesto, J., et al. (2020). World health organization recommendations on the treatment of drugresistant tuberculosis, 2020 update. Eur. Respir. J. doi: 10.1183/13993003.033002020 [Epub ahead of print].

Grossman, R. F., Hsueh, P. R., Gillespie, S. H., and Blasi, F. (2014). Communityacquired pneumonia and tuberculosis: differential diagnosis and the use of fluoroquinolones. Int. J. Infect. Dis. 18, 14-21. doi: 10.1016/j.ijid.2013.09.013

Guzman, J. D., Gupta, A., Bucar, F., Gibbons, S., and Bhakta, S. (2012). Antimycobacterials from natural sources: ancient times, antibiotic era and novel scaffolds. Front. Biosci. 17:1861-1881. doi: 10.2741/4024

Hu, Y. Q., Zhang, S., Zhao, F., Gao, C., Feng, L. S., Lv, Z. S., et al. (2017). Isoniazid derivatives and their anti-tubercular activity. Eur. J. Med. Chem. 133, 255-267. doi: 10.1016/j.ejmech.2017.04.002
Huynh, J., and Marais, B. J. (2019). Multidrug-resistant tuberculosis infection and disease in children: a review of new and repurposed drugs. Ther. Adv. Infect. Dis. 6, 1-16. doi: 10.1177/2049936119864737

Igarashi, M. (2017). Development of new antituberculosis drugs from natural products. Biosci. Biotechnol. Biochem. 81, 32-37. doi: 10.1080/09168451.2016. 1248369

Igarashi, M., Ishizaki, Y., and Takahashi, Y. (2018). New antituberculous drugs derived from natural products: current perspectives and issues in antituberculous drug development. J. Antibiot. 71, 15-25. doi: 10.1038/ja.2017. 126

Jansen, R. S., and Rhee, K. Y. (2017). Emerging approaches to tuberculosis drug development: at home in the metabolome. Trends Pharmacol. Sci. 38, 393-405. doi: 10.1016/j.tips.2017.01.005

Koul, A., Arnoult, E., Lounis, N., Guillemont, J., and Andries, K. (2011). The challenge of new drug discovery for tuberculosis. Nature 469, 483-490. doi: 10.1038/nature09657

Kumar, S., Bawa, S., and Gupta, H. (2009). Biological activities of quinoline derivatives. Mini. Rev. Med. Chem. 9, 1648-1654. doi: $10.2174 / 138955709791012247$

Luo, X., Zhou, X., Lin, X., Qin, X., Zhang, T., Wang, J., et al. (2017). Antituberculosis compounds from a deep-sea-derived fungus Aspergillus sp. SCSIO Ind09F01. Nat. Prod. Res. 31, 1958-1962. doi: 10.1080/14786419.2016. 1266353

Mishra, A., Mamidi, A. S., Rajmani, R. S., Ray, A., Roy, R., and Surolia, A. (2018). An allosteric inhibitor of Mycobacterium tuberculosis ArgJ: implications to a novel combinatorial therapy. EMBO Mol. Med. 10:e8038. doi: 10.15252/emmm. 201708038

Mosaei, H., Molodtsov, V., Kepplinger, B., Harbottle, J., Moon, C. W., Jeeves, R. E., et al. (2018). Mode of action of kanglemycin a, an ansamycin natural product that is active against rifampicin-resistant Mycobacterium tuberculosis. Mol. Cell. 72, 263-274. doi: 10.1016/j.molcel.2018.0 8.028

Nadav, E., Szilvia, B., Yoav, P., Shira, A., Jacob, G., Gal, R., et al. (2018). Structure of Type-I Mycobacterium tuberculosis fatty acid synthase at $3.3 \AA$ resolution. Nat. commun. 9:3886. doi: 10.1038/s41467-018-06440-6

Nunn, A. J., Phillips, P. P. J., Meredith, S. K., Chiang, C. Y., Conradie, F., Dalai, D., et al. (2019). A trial of a shorter regimen for rifampin-resistant tuberculosis. N. Engl. J. Med. 380, 1201-1213. doi: 10.1056/NEJMoa1811867

Peraman, R., Kuppusamy, R., Killi, S. K., and Reddy, Y. P. (2016). New conjugates of quinoxaline as potent antitubercular and antibacterial agents. Int. J. Med. Chem. 2016:6471352. doi: 10.1155/2016/6471352

Petrella, S., Capton, E., Raynal, B., Giffard, C., Thureau, A., Bonnete, F., et al. (2019). Overall structures of Mycobacterium tuberculosis DNA gyrase reveal the role of a Corynebacteriales GyrB-specific insert in ATPase activity. Structure 27, 579-589. doi: 10.1016/j.str.2019.01.004

Poushali, C., Sapna, B., Deepak, K., Bishan, D. R., Ashwani, K. (2021). Biofilm formation in the lung contributes to virulence and drug tolerance of Mycobacterium tuberculosis. Nat. Commun. 12:1606. doi: 10.1038/s41467-02121748-6

Pradhan, S., and Sinha, C. (2018). High throughput screening against pantothenate synthetase identifies amide inhibitors against Mycobacterium tuberculosis and Staphylococcus aureus. Silico. Pharmacol. 6:9. doi: 10.1007/s40203-018-0046-4

Rhastin, A. D. C., Amanda, R., Lujeko, K., Miriam, R., Chloe, L., Julia, F., et al. (2020). The genetic background modulates the evolution of fluoroquinoloneresistance in mycobacterium tuberculosis. Mol. Biol. Evol. 37, 195-207. doi: $10.1093 / \mathrm{molbev} / \mathrm{msz} 214$

Saba, N., Shruti, D., Sathya, N. N., Divya, A., Lakshya, V. S., Pradeep, K., et al. (2021). Compromised base excision repair pathway in Mycobacterium tuberculosis imparts superior adaptability in the host. PLoS Pathog. 17:e1009452. doi: 10.1371/journal.ppat.1009452

Sabine, E., Kyu, R., and Dirk, S. (2015). Mycobacterial genes essential for the pathogen's survival in the host. Immunol. Rev. 264, 319-326. doi: 10.1111/imr. 12256

Samiksha, G., Bhagwan, M., Erwin, M. J., Jos, G. W. K., Gokarna, R. G., Daan, J. T., et al. (2019). Levofloxacin pharmacokinetics, pharmacodynamics, and outcome in multidrug-resistant tuberculosis patients. Eur. Respir. J. 54:1802107. doi: 10.1183/13993003.02107-2018 
Sander, N. G., Samantha, L. S., and Annelies, V. R. (2020). Mechanisms of drug-induced tolerance in Mycobacterium tuberculosis. Clin. Microbiol. Rev. 34:e00141-20. doi: 10.1128/CMR.00141-20

Seung, K. J., Keshavjee, S., and Rich, M. L. (2015). Multidrug-resistant tuberculosis and extensively drug-resistant tuberculosis. Cold Spring Harb. Perspect. Med. 5:a017863. doi: 10.1101/cshperspect.a017863

Soutter, H. H., Centrella, P., Clark, M. A., Cuozzo, J. W., Dumelin, C. E., Guie, M. A., et al. (2016). Discovery of cofactor-specific, bactericidal Mycobacterium tuberculosis InhA inhibitors using DNA-encoded library technology. Proc. Natl. Acad. Sci. U.S.A. 113, E7880-E7889. doi: 10.1073/pnas.161097 8113

Takaki, K., Cosma, C. L., Troll, M. A., and Ramakrishnan, L. (2012). An in vivo platform for rapid high-throughput antitubercular drug discovery. Cell Rep. 2, 175-184. doi: 10.1016/j.celrep.2012.06.008

Tao, N. N., He, X. C., Zhang, X. X., Liu, Y., Yu, C. B., and Li, H. C. (2017). Drugresistant tuberculosis among children, China, 2006-2015. Emerg. Infect. Dis. 23, 1800-1805. doi: 10.3201/eid2311.170234

Tiago, B., Kathryn, O., Divya, T., Curtis, A. E., Shaun, W., Jenna, A., et al. (2019). Plasticity of the Mycobacterium tuberculosis respiratory chain and its impact on tuberculosis drug development. Nat. Commun. 10:4970. doi: 10.1038/s41467019-12956-2

Tweed, C. D., Dawson, R., Burger, D. A., Conradie, A., Crook, A. M., Mendel, C. M., et al. (2019). Bedaquiline, moxifloxacin, pretomanid, and pyrazinamide during the first 8 weeks of treatment of patients with drug-susceptible or drug-resistant pulmonary tuberculosis: a multicentre, open-label, partially randomised, phase 2b trial. Lancet Resp. Med. 7, 1048-1058. doi: 10.1016/S2213-2600(19)30366-2
World Health Organization (WHO). (2020). Global Tuberculosis Report. Available online at: https://www.who.int/teams/global-tuberculosis-programme/tbreports/global-tuberculosis-report-2020 (accessed January 30, 2021).

Yu, P., Zhao, J. Z., Feng, M. H., Wei, J., Yi, F. M., Ling, L., et al. (2017). In Vitro drug susceptibility of bedaquiline, delamanid, linezolid, clofazimine, moxifloxacin, and gatifloxacin against extensively drug resistant tuberculosis in Beijing, China. Antimicrob. Agents Chemother. 61, e00900-e00917. doi: 10. 1128/AAC.00900-17

Yuan, T., and Sampson, N. S. (2018). Hit generation in TB drug discovery: from genome to granuloma. Chem. Rev. 118, 1887-1916. doi: 10.1021/acs.chemrev. $7 \mathrm{~b} 00602$

Zuniga, E. S., Early, J., and Parish, T. (2015). The future for early-stage tuberculosis drug discovery. Fut. Microbiol. 10, 217-229. doi: 10.2217/fmb.1 4.125

Conflict of Interest: The authors declare that the research was conducted in the absence of any commercial or financial relationships that could be construed as a potential conflict of interest.

Copyright (c) 2021 Zhou, Yang, Zou, Rahman, Cao, Lei, Lai, Fu, Chen and Cao. This is an open-access article distributed under the terms of the Creative Commons Attribution License (CC BY). The use, distribution or reproduction in other forums is permitted, provided the original author(s) and the copyright owner(s) are credited and that the original publication in this journal is cited, in accordance with accepted academic practice. No use, distribution or reproduction is permitted which does not comply with these terms. 